\title{
Tracking unstable autoregressive sources over discrete memoryless channels
}

\section{Conference Paper}

Author(s):

Timo, Roy; Vellambi, Badri N.; Grant, Alex; Nguyen, Khoa D.

Publication date:

2016

Permanent link:

https://doi.org/10.3929/ethz-a-010645208

Rights / license:

In Copyright - Non-Commercial Use Permitted 


\section{Tracking Unstable Autoregressive Sources over Discrete Memoryless Channels}

\author{
Roy Timo \\ Technische Universität München \\ roy.timo@tum.de \\ Badri N. Vellambi \\ New Jersey Institute of Technology \\ badri.n.vellambi@ieee.org
}

\author{
Alex Grant \\ Cohda Wireless \\ alex.grant@cohdawireless.com
}

Khoa D. Nguyen

University of South Australia

khoa.nguyen@unisa.edu.au

\begin{abstract}
We consider the problem of tracking, in realtime, an unstable autoregressive (AR) source over a discrete memoryless channel (DMC). We present computable achievable bounds on the optimal tracking error for general DMCs, and we particularise these bounds to the binary erasure and packet erasure channels.
\end{abstract}

\section{Problem Setup}

Consider the scalar unstable $A R$ source

$$
W_{n}:=\lambda W_{n-1}+V_{n}, \quad n=1,2, \ldots,
$$

where $W_{0}=0, \lambda>1$ and $V_{1}, V_{2}, \ldots$ is iid and uniform on the real interval $\left[-v_{\max }, v_{\max }\right]$ for some positive $v_{\max }$. Suppose that a transmitter causally encodes and communicates (1) over a DMC to a receiver, and suppose that the receiver attempts to track (1) while operating with a finite decoding delay $\Delta$.

More formally, let us fix $\Delta$ to be any non-negative integer. Let $\mathcal{X}$ denote the DMC's input alphabet, $\mathcal{Y}$ its output alphabet and $T_{Y \mid X}: \mathcal{X} \rightarrow \mathcal{Y}$ its transition probabilities. A $\Delta$-code for tracking (1) consists of a sequence of mappings

$$
\left(F_{1}, G_{1}\right),\left(F_{2}, G_{2}\right), \ldots,
$$

where $F_{n}: \mathbb{R}^{n} \rightarrow \mathcal{X}$ and $G_{n}: \mathcal{Y}^{n+\Delta} \rightarrow \mathbb{R}$. The $n$-th channel symbol sent by the transmitter over the channel is

$$
X_{n}:=F_{n}\left(W_{1}, W_{2}, \ldots, W_{n}\right),
$$

The receiver estimates the $n$-th source symbol $W_{n}$ by

$$
\hat{W}_{n}:=g_{n}\left(Y_{1}, Y_{2}, \ldots, Y_{n+\Delta}\right) \text {. }
$$

Fix $\rho \geq 1$, and let

$$
\operatorname{ME}_{\rho}(\Delta, N):=\mathbb{E} \frac{1}{N} \sum_{n=1}^{N}\left|\hat{W}_{n}-W_{n}\right|^{\rho}
$$

denote the mean error in tracking. We call $N$ the blocklength, and we are interested in determining the following quantities.

Definition 1:

(i) The optimal $\mathrm{ME}_{\rho}$ for a given $\Delta$ and $N$,

$$
\operatorname{ME}_{\rho}^{*}(\Delta, N):=\inf _{\Delta \text {-codes }} \operatorname{ME}_{\rho}(\Delta, N) .
$$

(ii) The optimal $\mathrm{ME}_{\rho}$ for a given $\Delta$ and all $N$,

$$
\operatorname{ME}_{\rho}^{*}(\Delta):=\sup _{N \in\{1,2, \ldots\}} \operatorname{ME}_{\rho}^{*}(\Delta, N)
$$

The purpose of this paper is to report some useful achievable (upper) bounds on $\operatorname{ME}_{\rho}^{*}(\Delta, N)$ and $\operatorname{ME}_{\rho}^{*}(\Delta)$.
Remark 1: It can be shown that

$$
\sup _{N \in\{1,2, \ldots\}} \operatorname{ME}_{\rho}^{*}(\Delta, N)=\limsup _{N \rightarrow \infty} \operatorname{ME}_{\rho}^{*}(\Delta, N) .
$$

Consequently, $\operatorname{ME}_{\rho}^{*}(\Delta)$ measures the worst case tracking error as $N \rightarrow \infty$, and it is an appropriate engineering benchmark for problems where $N$ is large, varies or is otherwise unknown.

Remark 2: If $\Delta=0$, then for each $n=1,2, \ldots$ the receiver is required to output its estimate $\hat{W}_{n}$ of $W_{n}$ immediately upon observing the first $n$ channel outputs $Y_{1}, Y_{2}, \ldots, Y_{n}$. Here we have instantaneous communications in the sense that any "new information" in $W_{n}$ can only be communicated over the channel using $X_{n}$. If $\Delta>0$, then the receiver delays making an estimate of $W_{n}$ by $\Delta$ channel symbols. The transmitter now has more channel symbols from which to communicate each source symbol, and the receiver's estimates can therefore be improved. In essence, one can trade tracking reliability against decoding timeliness by varying $\Delta$.

Remark 3: The reader will have noticed that the unstable AR source in (1) does not fit within classical rate-distortion (RD) theory [1] (e.g., the AR source is non-stationary and nonergodic), and $\Delta$-codes and Definition 1 do not fit within the classical joint source-channel coding framework [2, Sec. 9.6]. Indeed, channels with the same capacity often behave quite differently under Definition 1, and the standard RD function, channel capacity and separation theorem offer little guidance on how to best approximate $\operatorname{ME}_{\rho}^{*}(\Delta, N)$ and $\operatorname{ME}_{\rho}^{*}(\Delta)$. Such behaviour has been observed throughout the realtime communications literature and is by no means unique to this paper, see, for example, the early work of [3]. A more complete literature review can be found in the longer version of this paper [4], and excellent literature reviews of realtime communications can be found in [5]-[7].

\section{EXAmples: Tracking OVER ERASURE Channels}

Let us first demonstrate the usefulness of this work by specialising the following achievable bound to the binary erasure channel (BEC) and packet erasure channel (PEC). (The extended paper [4] also considers the binary symmetric channel.) In this section, we restrict attention to mean absolute errors (MAEs); that is, we fix $\rho=1$ in (2).

Theorem 1:

$$
\left.\operatorname{ME}_{\rho}^{*}(\Delta, N)\right|_{\rho=1} \leq \min _{M}[\alpha(M)+\beta(M)],
$$




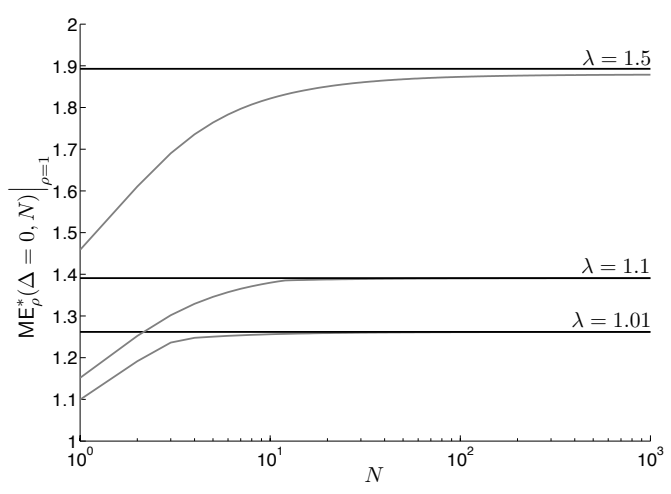

Fig. 1. The achievable bound on $\left.\operatorname{ME}_{\rho}^{*}(\Delta, N)\right|_{\rho=1}$ given in Proposition 1 (grey lines) and the achievable bound on $\left.\operatorname{ME}_{\rho}^{*}(\Delta)\right|_{\rho=1}$ given in Proposition 2 (black lines). The bounds are plotted for $\lambda=1.01,1.1$ and 1.5 with bandwidth expansion $\kappa=5$, zero decoding delay $\Delta=0$ and $v_{\max }=1$.

where the minimisation is over all integers $M \geq \max \{2, \lambda\}$,

$$
\alpha(M):=\frac{v_{\max }}{M-\lambda}
$$

and

$$
\begin{aligned}
\beta(M):=\frac{2 v_{\max }(M-1)}{N(M-\lambda)} \sum_{n=1}^{N} \sum_{k=1}^{n} \lambda^{n-k} \min \{1, & \\
& \left.\sum_{i=0}^{k-1} \tau_{M}(n+\Delta-i)\right\} .
\end{aligned}
$$

Theorem 1 follows from Lemma 1 and Theorem 3, which will be presented later in the paper. The upper bound depends on the channel law $T_{Y \mid X}$ via the function $\tau_{M}(\cdot)$, and we particularise $\tau_{M}(\cdot)$ to the BEC and PEC in the next two subsections ${ }^{1}$. Intuitively, $\alpha(M)$ can be understood as the quantisation error associated with discretising the source (it is the average error induced by an adaptive $M$ level scalar quantiser); and $\beta(M)$ can be understood as the channel distortion associated with streaming this $M$-level discrete approximation over the DMC $T_{Y \mid X}$. In general, a small $M$ will induce a large quantiser error and a small channel distortion, while a large $M$ will induce a small quantisation error and a large channel distortion. We now particularise $\tau_{M}(\cdot)$ to the BEC and PEC.

\section{A. Binary Erasure Channel (BEC)}

Suppose that the DMC consists of $\kappa$ independent BECs, each with the same erasure probability $0<\varepsilon<1$. Let

$$
\mathcal{X}=\{0,1\}^{\kappa} \text { and } \mathcal{Y}=\{0,1, \mathrm{e}\}^{\kappa},
$$

where e represents the erasure event; and

$T_{Y \mid X}(y \mid x)= \begin{cases}\varepsilon^{\mathrm{N}(y)}(1-\varepsilon)^{\kappa-\mathrm{N}(y)} & \text { if } x \text { and } y \text { agree on } \\ 0, & \text { unerased positions, } \\ \text { otherwise, }\end{cases}$

${ }^{1}$ We defer giving a formal definition of $\tau_{M}(\cdot)$ for general DMCs until Section IV, because this definition requires additional notation and ideas that are not needed for the BEC and PEC.

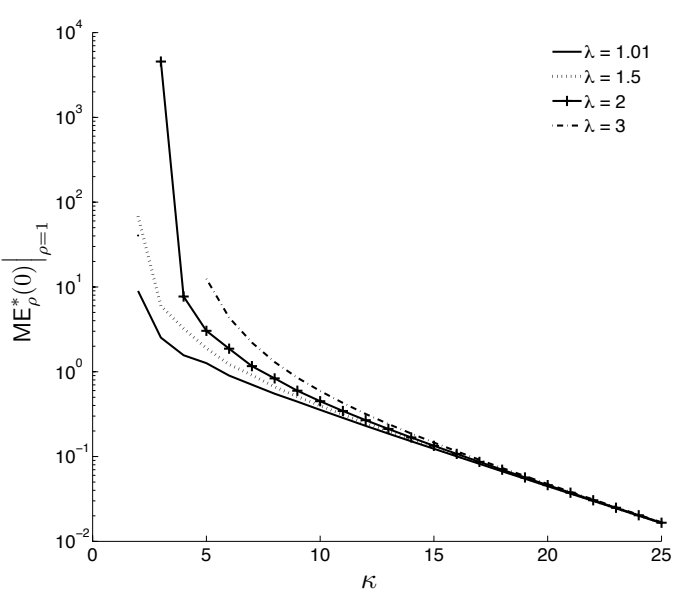

Fig. 2. The achievable bound in Proposition 2 for tracking (1) over a BEC with erasure probability $\epsilon=0.1$ and bandwidth expansion $\kappa$. The bound is plotted as a function of $\kappa$ for $\lambda=1.1,1.5,2$ and 3 with zero decoding delay $\Delta=0$ and $v_{\max }=1$.

where $\mathrm{N}(y)$ denotes the number of erased symbols in $y$. We call $\kappa$ the bandwidth expansion factor.

Proposition 1: For the BEC with erasure probability $\epsilon$ and bandwidth expansion $\kappa$, Theorem 1 holds with

$\tau_{M}(k)=\sum_{t=0}^{\kappa k}\left(\begin{array}{c}\kappa k \\ t\end{array}\right) \varepsilon^{t}(1-\varepsilon)^{\kappa k-t} 2^{-[(\kappa k-t)-k \log M-\log (M-1)]^{+}}$.

Proof: A proof can be found in [4, Appendix F-B].

A slight weakening of Proposition 1 yields the next proposition.

Proposition 2: For the BEC with erasure probability $\varepsilon$ and bandwidth expansion $\kappa$, we have

$$
\left.\operatorname{ME}_{\rho}^{*}(\Delta)\right|_{\rho=1} \leq \min _{M}\left[\alpha(M)+\gamma(M) 2^{-\Delta\left(\kappa \mathrm{R}_{0}(\varepsilon)-\log M\right)}\right],
$$

where

$$
\mathrm{R}_{0}(\varepsilon):=1-\log (1+\varepsilon)
$$

is the cutoff rate ${ }^{2}$ of the BEC,

$$
\begin{aligned}
\gamma(M):=\left(\frac{2 v_{\max }(M-1)^{2} M}{2^{\kappa \mathrm{R}_{0}(\varepsilon)}(M-\lambda)}\right) & \left(\frac{1}{1-M 2^{-\kappa \mathrm{R}_{0}(\varepsilon)}}\right) \\
& \left(\frac{1}{1-\lambda M 2^{-\kappa \mathrm{R}_{0}(\varepsilon)}}\right),
\end{aligned}
$$

and the minimisation is taken over all integers $M$ satisfying

$$
\max \{\lambda, 2\} \leq M<(1 / \lambda) 2^{\kappa \mathrm{R}_{0}(\varepsilon)} .
$$

Proof: A proof can be found in [4, Appendix F-B].

The bounds in Propositions 1 and 2 are plotted in Figure 1 as a function of the blocklength $N$ for three different values

${ }^{2}$ With a slight abuse of terminology, we use cutoff rate to refer to the standard $R_{0}$ parameter (see [12, Eqn. 14] or [11, p. 628]). The operational cutoff rate, which concerns the computational complexity of sequential decoding [12], does not appear to be related to this work. 


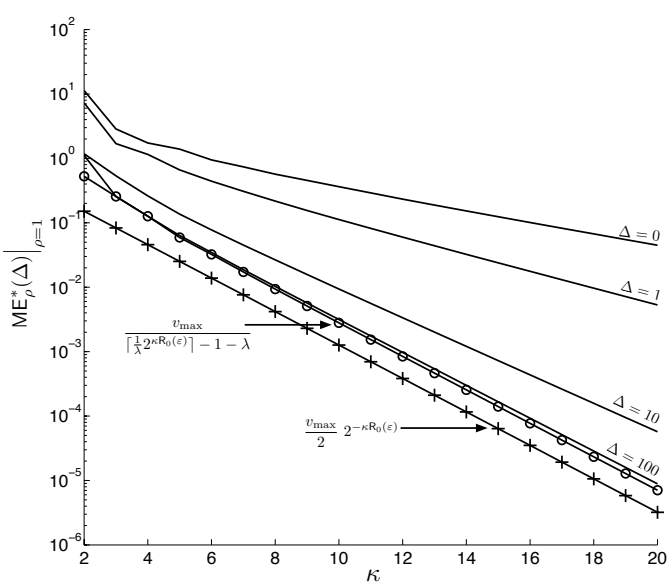

Fig. 3. The achievable bound in Proposition 2 for tracking (1) over a BEC with erasure probability $\epsilon=0.1$ and bandwidth expansion $\kappa$. The bound is plotted as a function of $\kappa$ for $\lambda=1.1,1.5,2$ and 3 with $\Delta=0$ and $v_{\max }=1$ fixed. The lower bound in Proposition 3 is also shown.

of $\lambda$. Figure 2 plots the bound in Proposition 2 as a function of the bandwidth expansion factor $\kappa$ for four different values of $\lambda$. Figure 3 illustrates the role of decoding delay $\Delta$ in Proposition 2. As $\Delta \rightarrow \infty$ the bound tends to

$$
\frac{v_{\max }}{\left\lceil\frac{1}{\lambda} 2^{\kappa \mathrm{R}_{0}(\varepsilon)}\right\rceil-1-\lambda} \approx \lambda v_{\max } 2^{-\kappa \mathrm{R}_{0}(\varepsilon)},
$$

so the cutoff rate $\mathrm{R}_{0}(\varepsilon)$ governs its asymptotic accuracy. To help get a feel for how useful Proposition 2 is, we now give a simple lower bound. This lower bound is shown in Figure 3.

Proposition 3: For the BEC with erasure probability $\varepsilon$, bandwidth expansion $\kappa$ and zero decoding delay $\Delta=0$,

$$
\left.\mathrm{ME}_{\rho}^{*}(0)\right|_{\rho=1} \geq \frac{v_{\max }}{2} 2^{-\kappa \mathrm{R}_{0}(\varepsilon)} .
$$

Proof: A proof can be found in [4, Appendix F-C].

\section{B. Packet Erasure Channel (PEC)}

Now imagine that the transmitter communicates with the receiver over a network that can be modelled ${ }^{3}$ by a $\kappa$ bit PEC

Fix $0<\varepsilon<1$, and let

$$
\mathcal{X}:=\{0,1\}^{\kappa} \text { and } \mathcal{Y}:=\{0,1\}^{\kappa} \cup\{\mathrm{e}\}
$$

and

$$
T_{Y \mid X}(y \mid x):=\left\{\begin{array}{cc}
1-\varepsilon & \text { if } y=x \\
\varepsilon & \text { if } y=\mathrm{e} \\
0 & \text { otherwise. }
\end{array}\right.
$$

Proposition 4: For the $\kappa$ bit PEC with erasure probability $\varepsilon$, Theorem 1 holds with

$$
\tau_{M}(k)=\sum_{t=0}^{k}\left(\begin{array}{l}
k \\
t
\end{array}\right) \varepsilon^{t}(1-\varepsilon)^{k-t} 2^{-[\kappa(k-t)-k \log M-\log (M-1)]^{+}} .
$$

${ }^{3}$ Here we assume that bit-level errors within a packet are handled on a link-by-link basis using physical layer error-correction techniques, and packets arrive at the receiver promptly or they are lost to, for example, congestion and buffer overflows.

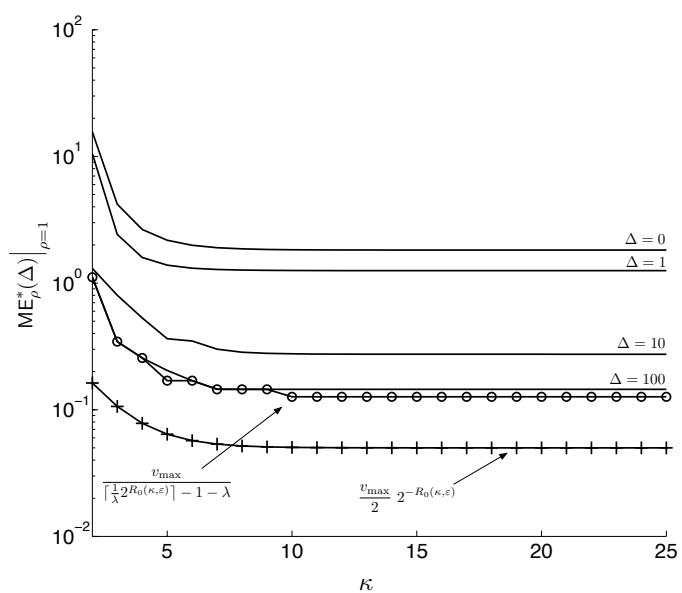

Fig. 4. The achievable bound on $\left.\operatorname{ME}_{\rho}^{*}(\Delta)\right|_{\rho=1}$ in Proposition 5 for tracking (1) with $\lambda=1.1$ and $v_{\max }=1$ over a $\kappa$ bit packet erasure channel with erasure probability $\varepsilon=0.1$. The bound is plotted as a function of $\kappa$ for decoding delays $\Delta=0,1,10$ and 100 . The lower bound in Proposition 6 is also shown.

Proof: A proof can be found in [4, Appendix G-A].

Proposition 5: For the $\kappa$ bit PEC with erasure probability $\varepsilon$,

$$
\left.\operatorname{ME}_{\rho}^{*}(\Delta)\right|_{\rho=1} \leq \min _{M}\left[\alpha(M)+\gamma(M) 2^{-\Delta\left(\mathrm{R}_{0}(\kappa, \varepsilon)-\log M\right)}\right],
$$

where

$$
\begin{aligned}
\gamma(M):=\left(\frac{2 v_{\max }(M-1)^{2} M}{2^{\mathrm{R}_{0}(\kappa, \varepsilon)}(M-\lambda)}\right) & \left(\frac{1}{1-M 2^{-\mathrm{R}_{0}(\kappa, \varepsilon)}}\right) \\
& \left(\frac{1}{1-\lambda M 2^{-\mathrm{R}_{0}(\kappa, \varepsilon)}}\right),
\end{aligned}
$$

the minimisation is over all integers $M$ satisfying

$$
\max \{2, \lambda\} \leq M<(1 / \lambda) 2^{\mathrm{R}_{0}(\kappa, \varepsilon)}
$$

and

$$
\mathrm{R}_{0}(\kappa, \varepsilon)=\kappa-\log \left(\varepsilon\left(2^{\kappa}-1\right)+1\right)
$$

is the cutoff rate of the $\kappa$-bit PEC.

Proof: A proof can be found in [4, Appendix G-B].

Proposition 6: For the $\kappa$ bit PEC with erasure probability $\varepsilon$ and zero decoding delay $(\Delta=0)$,

$$
\left.\operatorname{ME}_{\rho}^{*}(0)\right|_{\rho=1} \geq \frac{v_{\max }}{2} 2^{-\mathrm{R}_{0}(\kappa, \varepsilon)} .
$$

Proof: A proof can be found in [4, Appendix G-C].

Figure 4 illustrates the role of decoding delay in Proposition 5. Each curve exhibits a tracking 'error floor' because

$$
\lim _{\kappa \rightarrow \infty} \mathrm{R}_{0}(\kappa, \varepsilon)=-\log \varepsilon,
$$

so $M$ cannot grow without bound in $\kappa$. Interestingly, this error floor is intrinsic to the problem, because, for example, it also appears in Proposition 6. 


\section{Discussion}

Propositions 1 to 6 give computable upper and lower bounds for tracking (1) over two basic erasure channels. To the best of our knowledge, no computable bounds have appeared before in the literature ${ }^{4}$. Although we do not believe that the bounds are tight, we do believe that they provide useful performance benchmarks for practitioners in code design. For example, Figure 3 illustrates that the optimal MAE for the BSC improves exponentially fast with the bandwidth expansion factor $\kappa$; therefore, one might consider optimising $\kappa$ against the symbol error probability $\varepsilon$ on a system level. While, on the other hand, Figure 4 suggests that it does not make sense to use large packets over a PEC (unless one can decrease the erasure probability with increasing packet length). Finally, Theorem 1 is proved using a method that partially separates source quantisation from channel coding. Separation is often necessary in practice, and these bounds illustrate what one might achieve with such an approach.

\section{BOUNDS ON MAE VIA CHANNEL CODING WITH AN AR-DISTORTION FUNCTION}

The upper bounds on $\left.\operatorname{ME}_{\rho}^{*}(\Delta, N)\right|_{\rho=1}$ and $\left.\operatorname{ME}_{\rho}^{*}(\Delta)\right|_{\rho=1}$ presented in Section II all follow from the method outlined in this section, which can be applied to any DMC and $\rho \geq 1$. The key idea here will be to partially separate channel coding from quantisation, and to optimise the channel code with respect to an AR Hamming (ARH) distortion function that is determined by the source statistics. This section extends our earlier work [13] on streaming discrete AR sources with a MAE criterion to continuous AR sources with $\rho \geq 1$ in (2).

Arbitrarily fix an integer $M \geq 2$, and suppose that a discrete memoryless source (DMS) emits a sequence $U_{1}, U_{2}, \ldots$, of independent and uniformly distributed random variables on

$$
\mathcal{U}:=\{0, \ldots, M-1\} .
$$

An $(M, \Delta)$-channel code for streaming $U_{1}, U_{2}, \ldots$ over the DMC $T_{Y \mid X}$ consists of a sequence of mappings

$$
\left(f_{1}, g_{1}\right),\left(f_{2}, g_{2}\right), \ldots
$$

where

$$
f_{n}: \mathcal{U}^{n} \rightarrow \mathcal{X} \quad \text { and } \quad g_{n}: \mathcal{Y}^{n+\Delta} \rightarrow \mathcal{U}^{n}
$$

The $n$-th symbol sent over the channel by the transmitter is

$$
X_{n}:=f_{n}\left(U_{[1, n]}\right),
$$

where we let

$$
U_{[1, n]}=\left(U_{1}, U_{2}, \ldots, U_{n}\right)
$$

denote the first $n$ symbols output by the DMS (we will also employ this notation for other random vectors). The receiver estimates the first $n$ DMS symbols $U_{[1, n]}$ from the first $(n+\Delta)$ channel outputs $Y_{[1, n+\Delta]}=\left(Y_{1}, Y_{2}, \ldots, Y_{n+\Delta}\right)$ by

$$
\hat{U}_{[1, n]}^{(n)}:=g_{n}\left(Y_{[1, n+\Delta]}\right) \text {. }
$$

${ }^{4}$ Previous works, e.g., [7]-[10], appear to exclusively focus on checking whether or not certain tracking error measures are finite for a given channel.
A key point here is that the receiver initially estimates the first $n$ DMS symbols $U_{[1, n]}$ immediately upon observing the first $(n+\Delta)$-channel outputs. The receiver then revisits and (hopefully) improves this estimate as more channel outputs become available. The speed at which these estimates improve can be partially quantified by the following AR Hamming (ARH) distortion function. This distortion function will be our doorway to computable bounds on $\operatorname{ME}_{\rho}{ }^{*}(\Delta, N)$.

We define the ARH distortion between a source output $u_{[1, n]} \in \mathcal{U}^{n}$ and reconstruction $\hat{u}_{[1, n]} \in \mathcal{U}^{n}$ by

$$
\mathrm{d}_{n}\left(\hat{u}_{[1, n]}, u_{[1, n]}\right):=\sum_{k=1}^{n} \lambda^{n-k} \mathbb{1}\left\{\hat{u}_{[1, k]} \neq u_{[1, k]}\right\},
$$

where

$$
\mathbb{1}\left\{\hat{u}_{[1, k]} \neq u_{[1, k]}\right\}:= \begin{cases}1 & \text { if } \hat{u}_{[1, k]} \neq u_{[1, k]} \\ 0 & \text { otherwise. }\end{cases}
$$

For a given $(M, \Delta)$-code, let

$$
\mathrm{D}_{\rho, M}(\Delta, N):=\mathbb{E} \frac{1}{N} \sum_{n=1}^{N}\left(\mathrm{~d}_{n}\left(\hat{U}_{[1, n]}^{(n)}, U_{[1, n]}\right)\right)^{\rho} .
$$

We say that a distortion $D$ is $(\rho, M, \Delta, N)$-achievable if there exists an $(M, \Delta)$-channel code with $\mathrm{D}_{\rho, M}(\Delta, N) \leq D$. Let

$$
\mathrm{D}_{\rho, M}^{*}(\Delta, N):=\min \{D: D \text { is }(\rho, M, \Delta, N) \text {-achievable }\}
$$

denote the optimal ARH distortion for a given $\Delta$ and $N$. The key result of this section is the next lemma, which demonstrates that any achievable bound on $\mathrm{D}_{M}^{*}(\Delta, N)$ automatically gives an achievable bound on $\operatorname{ME}_{\rho}^{*}(\Delta, N)$.

Lemma 1: For every integer $M \geq \max \{\lambda, 2\}$, we have

$$
\begin{aligned}
& \operatorname{ME}_{\rho}^{*}(\Delta, N) \leq\left(\frac{2 v_{\max }}{M-\lambda}\right)^{\rho}\left(\frac{1}{2}+\right. \\
&\left.2^{\rho-1}(M-1)^{\rho} \mathrm{D}_{\rho, M}^{*}(\Delta, N)\right) .
\end{aligned}
$$

Proof: A proof can be found in [4, Appendix A].

IV. RAndom Coding Union Bound on $\mathrm{D}_{M}^{*}(\Delta, N)$

We now present an achievable bound on $\mathrm{D}_{M}^{*}(\Delta, N)$ that is motivated by the random coding union bound for block codes [14, Thm. 17]. We need the following notation. Given a pair of discrete random variables $(A, B)$ on $\mathcal{A} \times \mathcal{B}$ with joint pmf $P_{A B}(a, b)$ and marginals $P_{A}(a)$ and $P_{B}(b)$, the information density $\imath_{A ; B}: \mathcal{A} \times \mathcal{B} \rightarrow[-\infty, \infty]$ is

$$
\imath_{A ; B}(a ; b):=\log \frac{P_{A B}(a, b)}{P_{A}(a) P_{B}(b)} .
$$

Let $\mathcal{P}_{\mathcal{X}}$ denote the set of all pmfs on the channel input alphabet $\mathcal{X}$. For each $k \in\{1,2, \ldots\}$ and $P_{X} \in \mathcal{P}_{\mathcal{X}}$, let

$$
\begin{aligned}
\tau_{M}\left(P_{X}, k\right): & =\mathbb{E}[\min \{1, \\
& \left.\left.M^{k-1}(M-1) \zeta_{k}\left(X_{[1, k]}, Y_{[1, k]}, \tilde{X}_{[1, k]}\right)\right\}\right],
\end{aligned}
$$

where

$$
\left(X_{[1, k]}, Y_{[1, k]}, \tilde{X}_{[1, k]}\right)=\left(X_{1}, Y_{1}, \tilde{X}_{1}\right), \ldots,\left(X_{k}, Y_{k}, \tilde{X}_{k}\right)
$$


is a string of $k$ iid tuples

$$
(X, Y, \tilde{X}) \sim P_{X}(x) T_{Y \mid X}(y \mid x) P_{X}(\tilde{x})
$$

on $\mathcal{X} \times \mathcal{Y} \times \mathcal{X}$; the expectation in (3) is taken with respect to $\left(X_{[1, k]}, Y_{[1, k]}\right)$; and

$$
\begin{aligned}
\zeta_{k}\left(X_{[1, k]}, Y_{[1, k]}, \tilde{X}_{[1, k]}\right): & =\mathbb{P}\left[\sum_{i=1}^{k} \imath_{X ; Y}\left(\tilde{X}_{i} ; Y_{i}\right) \geq\right. \\
& \left.\sum_{i=1}^{k} \imath_{X ; Y}\left(X_{i} ; Y_{i}\right) \mid\left(X_{[1, k]}, Y_{[1, k]}\right)\right] .
\end{aligned}
$$

Theorem 2:

$$
\mathrm{D}_{\rho, M}^{*}(M, \Delta) \leq \mathrm{RCU}_{\rho, M}^{*}(\Delta, N)
$$

where

$$
\begin{aligned}
\operatorname{RCU}_{\rho, M}^{*}(\Delta, N):= & \inf _{P_{X} \in \mathcal{P}_{\mathcal{X}}} \frac{1}{N} \sum_{n=1}^{N}\left(\sum_{k=1}^{n}\left(\frac{\lambda^{n-k+1}-1}{\lambda-1}\right)^{\rho}\right. \\
& \left.\min \left\{1, \sum_{i=0}^{k-1} \tau_{M}\left(P_{X}, n+\Delta-i\right)\right\}\right)
\end{aligned}
$$

Proof: A proof can be found in [4, Appendix D].

Theorem 2 generalises our previous RCU achievable bound in [13, Thm. 1], which is summarised below in Theorem 3, from $\rho=1$ to arbitrary $\rho \geq 1$.

Theorem 3:

$$
\mathrm{D}_{M}^{*}(\Delta, N) \leq \mathrm{RCU}_{M}^{*}(\Delta, N)
$$

where

$$
\begin{aligned}
\operatorname{RCU}_{M}^{*}(\Delta, N):=\inf _{P_{X} \in \mathcal{P}_{\mathcal{X}}} \frac{1}{N} \sum_{n=1}^{N}\left(\sum_{k=1}^{n} \lambda^{n-k} \min \{1,\right. \\
\\
\left.\left.\sum_{i=0}^{k-1} \tau_{M}\left(P_{X}, n+\Delta-i\right)\right\}\right) .
\end{aligned}
$$

Theorem 3 is a little stronger than Theorem 2 for $\rho=1$, so we used this bound to prove Theorem 1 and the propositions in Section II.

\section{Boundedness of $\operatorname{ME}_{\rho}^{*}(\Delta)$ FOR GENERAL DMCs}

The achievable bounds on $\left.\operatorname{ME}_{\rho}^{*}(\Delta)\right|_{\rho=1}$ presented in Section II-A for the binary erasure and packet erasure channels followed by carefully bounding the function $\tau_{M}(\cdot)$ in Theorem 3. In this section, we consider arbitrary DMCs and give a sufficient condition for $\operatorname{ME}_{\rho}^{*}(\Delta)$ to be finite. To proceed, we first need the following definitions and notation.

Let us denote the capacity of the DMC $T_{Y \mid X}$ (in nats per channel use) by

$$
\mathrm{C}:=\max _{P_{X} \in \mathcal{P}_{\mathcal{X}}} I(X ; Y) .
$$

For rates $0 \leq R<\mathrm{C}$, the random-coding exponent of the DMC $T_{Y \mid X}$ is [2, p. 139]

$$
\mathrm{E}_{\mathrm{r}}(R):=\max _{\rho \in[0,1]} \max _{P_{X} \in \mathcal{P}_{\mathcal{X}}}\left[\mathrm{E}_{\mathrm{o}}\left(\rho, P_{X}\right)-\rho R\right],
$$

where

$\mathrm{E}_{\mathrm{o}}\left(\rho, P_{X}\right):=-\ln \left(\sum_{y \in \mathcal{Y}}\left(\sum_{x \in \mathcal{X}} P_{X}(x)\left(T_{Y \mid X}(y \mid x)\right)^{\frac{1}{1+\rho}}\right)^{1+\rho}\right)$.

Theorem 4:

$$
\begin{aligned}
& \sup _{N \in\{1,2, \ldots\}} \operatorname{RCU}_{M}^{*}(\Delta, N) \\
& \leq \inf _{R}\left(\frac{\left(1-e^{-R}\right)}{e^{\mathrm{E}_{\mathrm{r}}(R)}\left(1-e^{-\mathrm{E}_{\mathrm{r}}(R)}\right)\left(1-\lambda e^{-\mathrm{E}_{\mathrm{r}}(R)}\right)}\right) e^{-\Delta \mathrm{E}_{\mathrm{r}}(R)},
\end{aligned}
$$

where the infimum is taken over all $R>\ln 2$ such that $\ln \lambda<$ $\mathrm{E}_{\mathrm{r}}(R)$. If no such $R$ exists, then we take the bound to be infinite.

Proof: A proof can be found in [4, Appendix I].

Corollary 4.1: Suppose that the DMC satisfies $\mathrm{E}_{\mathrm{r}}(R)>0$ for all $0 \leq R<\mathrm{C}$ and $\mathrm{E}_{\mathrm{r}}(\mathrm{C})=0$. If

$$
\ln \lambda<\mathrm{E}_{\mathrm{r}}(\ln 2)<\mathrm{C},
$$

then $\left.\operatorname{ME}_{\rho}^{*}(\Delta)\right|_{\rho=1}$ is finite for all $\Delta \in\{0,1, \ldots\}$.

Proof: A proof can be found in [4, Appendix I].

\section{ACKNOWLEDGEMENTS}

This work was supported by the Alexander von Humboldt foundation and the ARC Grant DE12010016.

\section{REFERENCES}

[1] R. Gray, "Information rates of autoregressive processes," IEEE Trans. Inform. Theory, vol. 16, no. 4, pp. $412-421,1970$

[2] R. Gallager, Information Theory and Reliable Communication. John Wiley and Sons, Inc. New York, NY, USA, 1968.

[3] J. Walrand and P. Varaiya, "Optimal causal coding-decoding problems," IEEE Trans. Inform. Theory, vol. 29, no. 6, pp. $814-820,1983$.

[4] R. Timo, B. N. Vellambi, A. Grant, and K. D. Nguyen, "Tracking unstable autoregressive sources over discrete memoryless channels," preprint submitted to IEEE Trans. Inform. Theory, August, 2015. http://roytimo.wordpress.com/pub/

[5] D. Teneketzis, "On the structure of optimal real-time encoders and decoders in noisy communication," IEEE Trans. Inform. Theory, vol. 52, no. 9 , pp. $4017-4035,2006$

[6] A. Mahajan and D. Teneketzis, "Optimal design of sequential real-time communication systems," IEEE Trans. Inform. Theory, vol. 55, no. 11, pp. $5317-5338,2009$.

[7] S. Yüksel and T. Başar, Stochastic networked control systems. Birkhauser, 2013.

[8] A. Sahai, "Any-time capacity and a separation theorem for tracking unstable processes," in IEEE Intl. Symp. Inform. Theory, Italy, 2000.

[9] A. Sahai and S. Mitter, "The necessity and sufficiency of anytime capacity for stabilization of a linear system over a noisy communication link - Part I: scalar systems," IEEE Trans. Inform. Theory, vol. 52, no. 8, pp. 3369-3395, 2006.

[10] A. S. Matveev and A. V. Savkin, "An analogue of Shannon information theory for detection and stabilization via noisy discrete communication channels," SIAM J. Control Optim., vol. 46, no. 4, 2007.

[11] E. Arikan, "Channel combining and splitting for cutoff rate improvement," IEEE Trans. Inform. Theory, vol. 52, no. 2, pp. 628-639, 2006.

[12] - "A perspective on polar coding," preprint, 2015.

[13] R. Timo, A. Grant, and B. N. Vellambi, "Streaming with autoregressivehamming distortion for ultra short-delay communications," in IEEE Intl. Symp. Inform. Theory, Honolulu, USA, 2014.

[14] Y. Polyanskiy, "Channel coding: non-asymptotic fundamental limits," $\mathrm{PhD}$ Thesis, Princeton University, 2010. 